\title{
The Age-Specific Features and Clinical Significance of NRF2 and MAPK IO Expression in HCC Patients
}

\author{
Manar Atyah',*, Chenhao Zhou (1) '*, Qiang Zhou', Wanyong Chen', Jialei Weng', Pengcheng Wang', \\ Yi Shi ${ }^{2}$, Qiongzhu Dong ${ }^{3,4}$, Ning Ren ${ }^{1,3}$ \\ 'Department of Liver Surgery, Liver Cancer Institute, Zhongshan Hospital, and Key Laboratory of Carcinogenesis and Cancer Invasion (Ministry of \\ Education), Fudan University, Shanghai, 200032, People's Republic of China; ${ }^{2}$ Biomedical Research Centre, Zhongshan Hospital, Fudan University, \\ Shanghai, 200032, People's Republic of China; ${ }^{3}$ Institute of Fudan Minhang Academic Health System, and Key Laboratory of Whole-period Monitoring \\ and Precise Intervention of Digestive Cancer (SMHC), Minhang Hospital \& AHS, Fudan University, Shanghai, 20I 199, People's Republic of China; \\ ${ }^{4}$ Institutes of Biomedical Sciences, Fudan University, Shanghai, 200032, People's Republic of China
}

Correspondence: Ning Ren, Department of Liver Surgery, Liver Cancer Institute, Zhongshan Hospital, and Key Laboratory of Carcinogenesis and Cancer Invasion (Ministry of Education), Fudan University, Shanghai, 200032, People's Republic of China, Tel +86-2I-6404l990, Email ren.ning@zs-hospital.sh.cn

Qiongzhu Dong, Institute of Fudan Minhang Academic Health System, and Key Laboratory of Whole-period Monitoring and Precise Intervention of Digestive Cancer (SMHC), Minhang Hospital \& AHS, Fudan University, Shanghai, 20I 199, People’s Republic of China, Tel +86-2I- 64923400, Email qzhdong@fudan.edu.cn

*These authors contributed equally to this work

Background: Nuclear factor (erythroid-derived 2)-like 2 (NRF2) functions decline with age; however, cancer cells can hijack its pathways to ensure survival and aggressiveness. Yet, the role of NRF2 in hepatocellular carcinoma (HCC) is rarely investigated in an age-specific manner. This study investigates the expression of NRF2 and its activator (MAPK10) in different age groups of HCC patients, in addition to the age-specific features of NRF2 and MAPK10 interaction and their clinical significance.

Methods: Tumor and near-tumor tissue samples of $181 \mathrm{HCC}$ patients were used to complete a protein expression analysis of NRF2 and MAPK10. Patients' survival and clinical data were collected for clinical analysis. Global databases (TCGA, ICGC) were used to collect MAPK10 genetic mutation and mRNA expression data in patients with HCC, colorectal, stomach, and pancreatic cancers.

Results: Our findings revealed an increase in NRF2 protein expression but only in younger HCC patients, along with a decline in MAPK10 ability to activate NRF2 in older patients. We also found an increased MAPK10 genetic mutation rate and decreased mRNA expression in older patients. Low MAPK10 and NRF2 expression levels were associated with shorter survival and poorer prognosis due to positive correlation with microvascular invasion, tumor thrombus, elevated AFP levels, and larger tumor size.

Conclusion: NRF2 expression and oxidative stress mechanism in HCC patients are influenced by age. This magnifies the need to consider patients' age in treatment strategies and guidelines and re-evaluates the application of studies' age-standardized findings in older patients who are usually excluded from relevant research.

Keywords: NRF2, MAPK10, hepatocellular carcinoma, oxidative stress, aging

\section{Introduction}

The aging of population is a serious problem faced all over the world. The number of elderly people ( $\geq 80$ years) is estimated to triple by 2050 (compared to 2019). ${ }^{1}$ Therefore, countries are constantly faced with the challenges and changes this phenomenon brings to societies. Such changes in age structure directly influenced the incidence of cancer. In 2018, a global estimation reported that $13 \%$ of total cancer cases were recorded in elderly patients ( $\geq 80$ years), and expected the percentage to reach $20.5 \%$ by $2050 .^{2}$ To cope with the changes, healthcare systems have to enhance and customize services to adapt to the new requirements, and should consider additional factors like age-related complications, comorbidity, and shorter life expectancy. ${ }^{2}$ However, older patients are usually excluded from clinical trials and related studies. That created a lack of age-specific researches and left the age-specific risks and benefits of certain treatments widely unknown. ${ }^{3}$ 
In the case of primary liver cancer, a globally common cancer which ranks sixth in incidence and forth in cancermortality, ${ }^{4} 60$ years is the approximate age of diagnosis. ${ }^{5}$ Within a period of 25 years (1990-2015), the incidence of liver cancer had a $75 \%$ increase, $55 \%$ of which was considered age-influenced. ${ }^{6}$

Cellular aging is known for certain "hallmarks" such as genetic and epigenetic instabilities, decreased ability of cellular functioning, and compromised cellular interaction. ${ }^{7}$ Many mechanisms were suggested to explain the process of aging, one of which is the oxidative stress accumulation theory. Normally, cellular processes produce reactive oxygen species (ROS). Such production is tightly controlled to ensure cellular survival and normal functioning. However, when the reduction of ROS is compromised, a cellular accumulation starts to occur, which eventually leads to cellular dysfunction, genomic alterations, and finally cellular apoptosis. ${ }^{8}$ The previous process is strictly controlled by a complicated cellular antioxidant system, which involves several mechanisms, pathways, and factors such as nuclear factor (erythroid-derived 2)-like 2 (NRF2).

NRF2 is a transcription factor that interacts with many genes and affects the transcription of molecules involved in redox regulation, cellular metabolism, genomic repairing, and cellular survival. ${ }^{9-12}$ In general, NRF2 protects normal cells from carcinogenic changes due to its involvement in antioxidation and immune cellular functions. ${ }^{13-16}$ The accumulation of ROS stimulates MAPK10 (Mitogen-activated protein kinase 10) which activates the expression and translocation of NRF2. Once activated and translocated to the nucleus, NRF2 regulates the expression of antioxidant response elements (AREs). ${ }^{17-21}$ However, NRF2 expression and functions decline with age, leading to higher oxidative stress levels, accumulation of genetic alterations, and compromised cellular functioning. ${ }^{22,23}$ On the other hand, studies found that cancer cells can hijack NRF2 pathways $^{24}$ and maintain high activation levels (NRF2 addiction) to ensure a better survival, stronger therapeutic resistance, and more aggressive behavior. ${ }^{13}$ Such an activation of NRF2 has been proposed in many studies ${ }^{13,25-27}$ as a prognostic factor for cancer patients.

Similarly, NRF2 provides protection in normal liver cells but can also be hijacked by cells of hepatocellular carcinoma (HCC) (the most common type of liver cancer). ${ }^{28}$ However, conflicting findings have been reported regarding the exact role of NRF2 in HCC. Cheng et al ${ }^{29}$ observed a decrease in NRF2 expression in HCC, accompanied by an increased expression of its negative regulator KEAP1 (kelch-like ECH-associated protein 1). On the other hand, studies, like Zhang et al, ${ }^{30}$ found NRF2 to be highly expressed in HCC and observed a correlation between its levels and HCC size, differentiation, and metastasis; therefore, NRF2 high expression was suggested as an indication of poor prognosis.

Although the role of NRF2 in HCC has been addressed, this topic is rarely investigated in an age-specific manner, and a huge gap still exists in our knowledge of NRF2 role and mechanisms in elderly HCC patients. In this study, we investigated the differences in expression levels of NRF2 and its activator (MAPK10) among different age groups of HCC patients. In addition, we explored the age-specific features of NRF2 and MAPK10 interaction and evaluated their clinical significance.

\section{Patients and Methods}

\section{Patients}

Different sections of this study included different groups of patients:

A total of 181 patients 1) with pathological diagnosis of HCC and 2) who underwent surgical resection (hepatectomy) between 2009 and 2010 in the department of liver surgery and transplantation, Zhongshan Hospital, Fudan University were included in the immunohistochemistry (IHC) analysis of NRF2 and MAPK10 protein expression. Tumor tissue and near-tumor tissue samples (NRF2: 181 and 167 samples, MAPK10: 181 and 166 samples, respectively) were provided by the department along with patients' relevant data. Near-tumor tissue samples were collected further than $2 \mathrm{~cm}$ away from tumor margin. For patients' survival analysis, data of 180 (out of 181) patients were available and included. Written informed consent was obtained from all included patients and the study was approved by the Ethics Committee of Zhongshan Hospital, Fudan University and completed in accordance with the Helsinki declaration. After the collection of samples and data, IHC analysis was carried and the expression of the two genes was evaluated. 
For patients included in the analysis of genetic mutation rates, global databases (TCGA (https://www.cancer.gov/ tcga), ICGC (https://dcc.icgc.org/) ${ }^{31,32}$ ) were used to collect relevant data. For TCGA data collection, cBioPortal $^{33,34}$ platform was used. Collected data included 1025 HCC patients (USA: TCGA PanCancer Atlas /355 patients, China: LICA-CN (https://dcc.icgc.org/projects/LICA-CN)/163, France: LICA-FR (https://dcc.icgc.org/projects/LICA-FR) /249, Japan: LIRI-JP (https://dcc.icgc.org/projects/LIRI-JP)/258). To determine the significance of results, HCC findings were compared to findings of other groups of patients with colorectal cancer (a total of 853: USA: TCGA PanCancer Atlas /532, China: COCA-CN (https://dcc.icgc.org/projects/COCA-CN) /321), stomach cancer (a total of 555: USA: TCGA PanCancer Atlas /432, China: GACA-CN (https://dcc.icgc.org/projects/GACA-CN) /123), and pancreatic cancer (a total of 795: USA: TCGA PanCancer Atlas /179, Australia: PACA-AU (https://dcc. icgc.org/projects/PACA-AU) /383, Canada: PACA-CA (https://dcc.icgc.org/projects/PACA-CA) /233). All used data and projects are available online with no embargo or limitation of use (https://docs.icgc.org/portal/publication) (https://www.cancer.gov/about-nci/organization/ccg/research/structural-genomics/tcga/using-tcga/citing-tcga).

For the analysis of mRNA expression patterns, TCGA database and human protein atlas ${ }^{35,36}$ were used to obtain relevant data. In total, 362 HCC patients were included.

\section{Analysis of Patients' Survival and Clinical Characteristics}

For the analysis of patients' survival, overall survival (OS) and disease-free survival (DFS) data of 180 patients were obtained and included. OS was defined as the time between surgery and date of death or last follow-up while DFS was defined as the time between surgery and date of recurrence or last follow-up. Five-year and two-year survival rates were calculated to represent long-term and short-term survival rates, respectively.

As for the clinical feature analysis, data of 181 patients were included (unless specified otherwise). Characteristics such as patients' gender, liver cirrhosis status, tumor differentiation and encapsulation, number and size of lesions, presence of microvascular invasion (MVI), tumor thrombus, BCLC grade, and levels of HBsAg (+:>1.0 COI), AFP $(+:>20 \mathrm{ng} / \mathrm{mL})$, CEA (+:>5 ng/mL), CA19-9 (+:>36 U/mL), ALT (+: >40 U/L), AST (+: >35 U/L), ALP (+: >135 U/L), GGT (+: >45 U/L), TB $(+:>20.4 \mu \mathrm{mol} / \mathrm{L})$, and $\mathrm{DB}(+:>6.8 \mu \mathrm{mol} / \mathrm{L})$ were included.

\section{Immunohistochemistry (IHC)}

All obtained tissue samples were embedded in paraffin; thus, descending ethanol gradient was used to deparaffinize and rehydrate samples. Hydrogen peroxide (3\%) was then used for 10 minutes to block peroxidase activities. Antigen epitope retrieval was induced by placing samples in $0.1 \mathrm{M}$ citrate buffer and heating them in a microwave for 8 minutes. Then, samples were treated with $10 \%$ goat serum and incubated (at $4{ }^{\circ} \mathrm{C}$ temperature) with primary antibody overnight. Samples were then incubated with secondary antibody (at room temperature) for 30 minutes. Afterwards, 3,3'-diaminobenzidine was used to complete the staining of samples and visualize the outcome. Staining assessment was completed separately by two different doctors based on the extent (using a scale from 0 to $4(0 \%, 1-5 \%, 6-25 \%, 26-75 \%, 76-100 \%)$ ) and intensity (using a scale from 0 to 2 ( 0 : negative, 1: weak, 2: strong)). Nuclear staining was considered for NRF2, while cytoplasmic staining was considered for MAPK10. Final scores (extent $\times$ intensity) ranged from 0 to 8 and were divided into low expression group (0-4) and high expression group (6-8). ${ }^{37}$

\section{MAPK I0 Genetic Mutation Rates and mRNA Expression Patterns}

Mutation rates (\%) of MAPK10 were calculated in all four cancers, then calculated based on age groups in each cancer ( $\leq 19$ y, 20-39 y, 40-59 y, 69-79 y, $\geq 80$ y). Significant differences at the age of 60y were then separately calculated in each cancer. Afterwards, results from colorectal cancer, stomach cancer, and pancreatic cancer groups were combined to form one compare group. The significance of mutation rates was then determined by comparing rates in HCC age groups $(<60 \mathrm{y}$ vs $\geq 60 \mathrm{y})$ and rates in older patients $(\geq 60 \mathrm{y})$ (HCC vs compare group).

As mentioned, TCGA database and human protein atlas were used for MAPK10 mRNA expression analysis. First, significant expression cut-off (FPKM) was determined based on patients' survival data. After that, patients were divided into two age groups ( $\leq 59 \mathrm{y}, \mathrm{N}=165 \mathrm{vs} \geq 60 \mathrm{y}, \mathrm{N}=197$ ) and each group was divided (according to expression cut-off) into 
high and low expression subgroups. Relation between mRNA expression and age was then evaluated based on expression rates in each subgroup.

\section{Statistical Analysis}

SPSS software (21.0, IBM Corp.) and STATA software (13.0, College Station, Texas 77845, USA) were used to complete the statistical analysis of this study. Continuous variables and categorical variables were presented as means \pm standard deviations (SD) and frequencies (\%), respectively. Chi square test $\left(\mathrm{X}^{2}\right)$ was used to complete groups and subgroups comparisons (categorical variables). Kaplan-Meier's (K-M) method, the Log rank test, and COX regression analysis were used to analyze OS and DFS. P values less than 0.05 were considered statistically significant.

\section{Results}

\section{NRF2 and MAPKI0 Protein Expression}

The first section of this study aimed to analyze NRF2 expression levels in HCC patients (using IHC) and investigate differences among age groups ( $\leq 59 \mathrm{y}$ vs $\geq 60 \mathrm{y}$ ). In total, 181 patients were included in this analysis (181 cancer tissue and 167 near-tumor tissue samples). All samples were divided into high and low expression groups based on IHC assessment. Figure 1 shows representative samples of IHC staining of nucleus and cytoplasm. When comparing HCC to near-tumor tissues, we found that $29.83 \%$ of all HCC samples had high NRF2 expression, compared to only $11.38 \%$ in near-tumor samples $(\mathrm{P}<0.001)$. A further age-based analysis showed similar results in younger patients $(\leq 59 \mathrm{y})$. In $\mathrm{HCC}$ group, $35.2 \%$ of samples showed high NRF2 expression, compared to $12.07 \%$ in near-tumor samples $(\mathrm{P}<0.001)$. On the other hand, no statistically significant results were found in older patients ( $\geq 60 \mathrm{y})(\mathrm{P}=0.231)$, and high expression rates were $17.86 \%$ and $9.8 \%$ in HCC and near-tumor groups, respectively.

Based on the previous results, we further analyzed the expression levels of MAPK10. Similar to NRF2 analysis, 181 patients were included (181 cancer samples and 166 near-tumor samples). Differences between age groups ( $\leq 59 \mathrm{y}$ vs $\geq 60 \mathrm{y}$ ) were also investigated. Our finding showed that only $16.57 \%$ of all HCC samples had high MAPK10 expression, compared to $56.63 \%$ in near-tumor samples $(\mathrm{P}<0.001)$. Similar statistically significant results were found in both age groups. In younger patients ( $\leq 59 \mathrm{y}$ ), only $16 \%$ of HCC samples had high MAPK10 expression compared to $53.91 \%$ in near-tumor
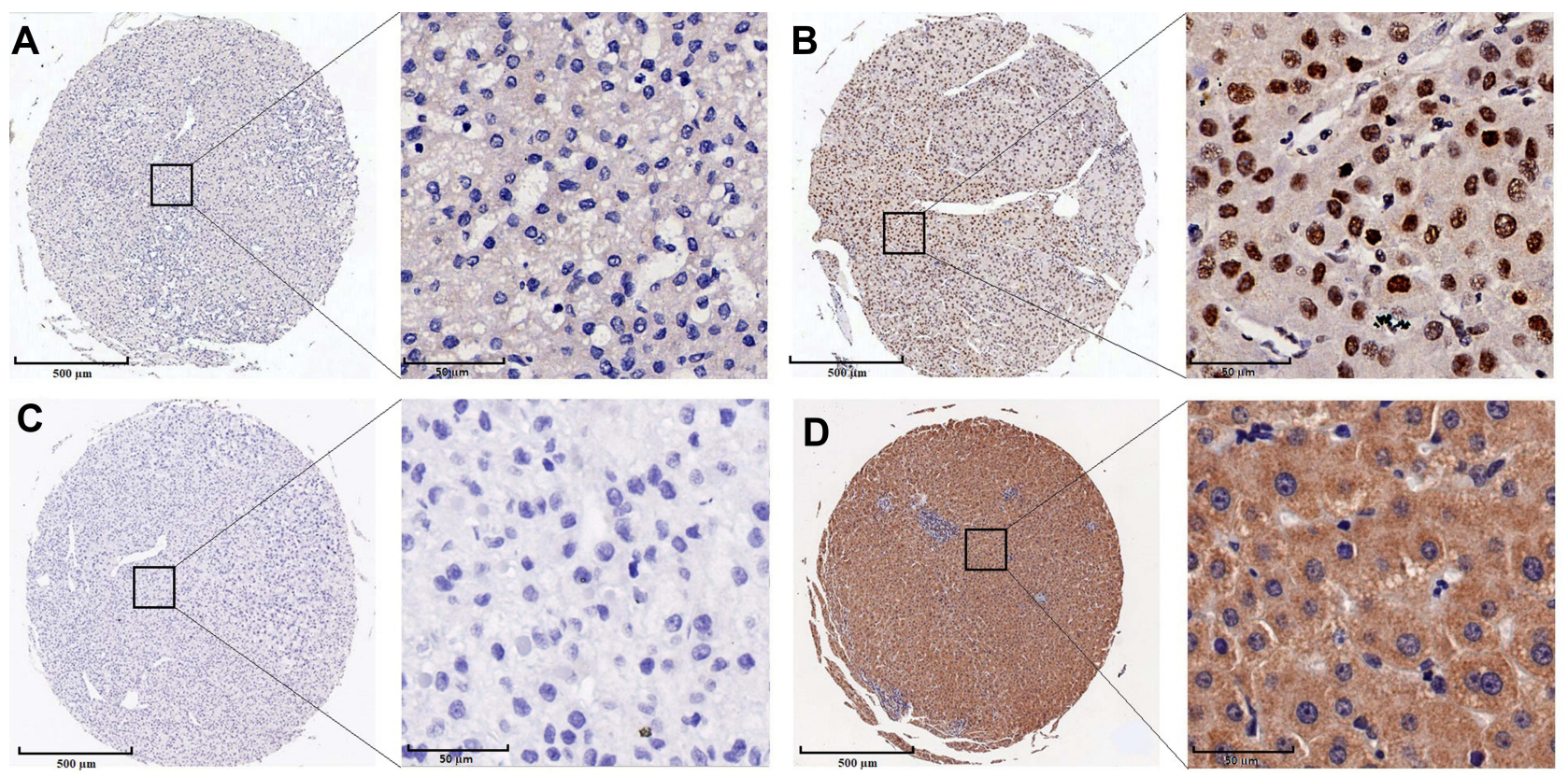

Figure I Representative samples of immunohistochemical staining of nucleus (A) weak staining; (B) strong staining) and cytoplasm (C) weak staining; (D) strong staining). 
samples $(\mathrm{P}<0.001)$. As for older patients $(\geq 60 \mathrm{y})$, the rates were $17.86 \%$ of $\mathrm{HCC}$ samples and $62.74 \%$ of near-tumor samples $(\mathrm{P}<0.001)$.

We also analyzed the relation between NRF2 and MAPK10 expression, findings showed 50\% of high MAPK10 HCC samples had high NRF2 expression, compared to only $25.83 \%$ in low MAPK10 HCC samples ( $\mathrm{P}=0.008)$. Similar results were found in the two age groups; however, only results from younger age group were statistically significant. In HCC samples of younger patients ( $\leq 59 \mathrm{y}$ ), high NRF2 expression was found in $60 \%$ of high MAPK10 expression group and $30.48 \%$ of low MAPK10 expression group ( $\mathrm{P}=0.011$ ), while the rates in older patients' group ( $\geq 60 \mathrm{y}$ ) were $30.00 \%$ and $15.22 \%$ respectively $(\mathrm{P}=0.515)$.

Results of near-tumor samples showed no significant differences (all patients: high MAPK10 12.77\% vs low MAPK10 9.72\% ( $\mathrm{P}=0.542)$; $\leq 59$ y: high MAPK10 12.90\% vs low MAPK10 11.32\% ( $\mathrm{P}=0.796)$; $\geq 60$ y: high MAPK10 $12.50 \%$ vs low MAPK10 5.26\% (P=0.639)).

To analyze from a different angle, we compared results of patients with high MAPK10 expression. Our findings showed that $50 \%$ of HCC samples had high NRF2 expression, while the percentage dropped to only $12.77 \%$ in neartumor samples $(\mathrm{P}<0.001)$. As for the two age groups, significant results $(\mathrm{P}<0.001)$ were found only in younger patients (high NRF2 expression in $60 \%$ of HCC samples vs $12.9 \%$ of near-tumor samples), while older patients' group had no significant value $(\mathrm{P}=0.418)(30 \%$ and $12.5 \%$, respectively).

\section{MAPK I0 Genetic Mutation Rates and mRNA Expression Patterns}

As mentioned, MAPK10 genetic mutation analysis was carried in four cancers and five age groups. Our results showed higher mutation rates in pancreatic cancer and HCC groups (28.43\% and $25.37 \%$, respectively) compared to stomach cancer $(15.86 \%)$ and colorectal cancer $(5.86 \%)$ groups. A gradual increase in mutation rates with age was only observed in HCC group and an increase of $21.23 \%$ was observed between HCC patients younger than 60y compared to older patients $(\geq 60 \mathrm{y})$ (group rates were $12.94 \%$ and $34.17 \%$, respectively) $(\mathrm{P}<0.001)$. On the other hand, a gradual decrease with age was observed in colorectal cancer group. Patients younger than $40 \mathrm{y}$ had a mutation rate of $19.51 \%$ compared to $5.17 \%$ in older patients $(\mathrm{P}=0.001)$. Rates from colorectal cancer, stomach cancer, and pancreatic cancer groups were then combined to form one compare group. The results in this group showed that patients younger than $60 \mathrm{y}$ had a $16.81 \%$ mutation rate compared to $16.39 \%$ in older patients $(\geq 60 \mathrm{y})(\mathrm{P}=0.803)$. When comparing results in older patients $(\geq 60 \mathrm{y})$, we found that mutation rates were higher in HCC patients than in compare group with a difference of $17.78 \%$ ( $\mathrm{P}<0.001)$. Detailed mutation rates in all four cancers and five age groups are presented in Table 1.

The analysis of MAPK10 mRNA expression was based on data obtained from TCGA database and human protein atlas. HCC data showed that MAPK10 expression higher than 0.09 FPKM was beneficial for patients' survival $(\mathrm{P}=0.046)$. Therefore, this expression value was chosen as a cut-off for high and low expression subgroups of each age group ( $\leq 59 \mathrm{y}, \mathrm{N}=165 \mathrm{vs} \geq 60 \mathrm{y}, \mathrm{N}=197)$. Our age group comparison showed that $67.27 \%$ of younger patients $(\leq 59)$ had high mRNA expression compared to $51.78 \%$ in older patients $(\geq 60 \mathrm{y})(\mathrm{P}=0.003)$.

Table I Detailed MAPKIO Genetic Mutation Rates in Different Cancers (HCC, Colorectal Cancer, Stomach Cancer, and Pancreatic Cancer) and Age Groups

\begin{tabular}{|c|c|c|c|c|c|c|}
\hline Cancer & $\begin{array}{c}\text { Group } \leq 19 y \\
(n / N) *(\%)\end{array}$ & $\begin{array}{c}\text { Group 20-39 y } \\
(n / N) *(\%)\end{array}$ & $\begin{array}{c}\text { Group 40-59 y } \\
(n / N) *(\%)\end{array}$ & $\begin{array}{c}\text { Group } 60-79 \text { y } \\
(n / N) *(\%)\end{array}$ & $\begin{array}{c}\text { Group } \geq 80 \text { y } \\
(\mathbf{n} / \mathbf{N})^{*}(\%)\end{array}$ & $\begin{array}{l}\text { All Patients } \\
(n / N) *(\%)\end{array}$ \\
\hline $\mathrm{HCC}$ & $(0 / 3)(0)$ & $(9 / 69)(13.04)$ & $(46 / 353)(13.03)$ & $(184 / 555)(33.15)$ & $(21 / 45)(46.67)$ & (260/1025) (25.37) \\
\hline Colorectal cancer & $(0 / 0)(0)$ & $(8 / 4 I)(19.5 I)$ & $(22 / 276)(7.97)$ & $(16 / 434)(3.69)$ & $(4 / 102)(3.92)$ & $(50 / 853)(5.86)$ \\
\hline Stomach cancer & $(0 / 0)(0)$ & (I/9) (II.II) & $(38 / 178)(21.35)$ & $(43 / 330)(13.03)$ & $(6 / 38)(15.79)$ & $(88 / 555)(15.86)$ \\
\hline Pancreatic cancer & $(0 / 0)(0)$ & $(2 / 8)(25)$ & $(50 / 208)(24.04)$ & $(156 / 518)(30.12)$ & $(|8 / 6|)(29.5 I)$ & $(226 / 795)(28.43)$ \\
\hline
\end{tabular}

Note: $*_{n}=$ number of cases with mutations, $\mathrm{N}=$ total number of cases. 


\section{MAPKIO and NRF2 Influence on Survival and Clinical Characteristics}

We started this section of the study by analyzing the influence of MAPK10 and NRF2 on long-term (5y) OS and DFS. Our findings showed that high MAPK10 expression was beneficial for both long-term OS (HR $=0.425$; 95\% CI, 0.214 0.845) $(\mathrm{P}=0.015)$ and DFS $(\mathrm{HR}=0.573 ; 95 \% \mathrm{CI}, 0.339-0.969)(\mathrm{P}=0.038)$. NRF2 influence on long-term survival was statistically insignificant (OS $\mathrm{P}=0.598$, DFS $\mathrm{P}=0.843$ ).

K-M analysis showed that high MAPK10 expression prolonged patients' $\mathrm{OS}(\mathrm{P}=0.011)$ and $\mathrm{DFS}(\mathrm{P}=0.035)$ for 10 months (high expression group: OS:49.244 \pm 3.53 , DFS: $40.537 \pm 3.969$, low expression group OS: $38.735 \pm 1.856$, DFS: $30.525 \pm 1.861$ ) (Figure 2A and B). The influence of NRF2 on short-term survival (2y) was also investigated. K-M analysis showed that high NRF2 expression prolonged short-term OS for 2 months (high expression group: $21.469 \pm 0.738$, low expression group: 19.233 $\pm 0.658, \mathrm{P}=0.047$ ). The influence on short-term DFS was statistically insignificant (high expression group: 18.306 \pm 1.041 , low expression group: $17.102 \pm 0.775, \mathrm{P}=0.712$ ) (Figure $2 \mathrm{C}$ and $\mathrm{D}$ ). A further $\mathrm{K}-\mathrm{M}$ analysis also showed that patients with high expression of both MAPK10 and NRF2 had prolonged long-term OS (13 months) compared to low expression group (high MAPK10 and NRF2 group: 51.597 \pm 4.338 , low MAPK10 and NRF2 group: 38.397 \pm 2.222 ) ( $\mathrm{P}=0.037$ ) (Figure 2E). No significant difference was found between patients with high NRF2 expression alone (39.7 \pm 3.385$)$ and those with low MAPK10 and NRF2 expression (38.397 \pm 2.222$)(\mathrm{P}=0.69)$.

The influence of expression levels on clinical characteristics was also investigated. Our findings revealed that low MAPK10 expression was significantly associated with higher rates of MVI $(\mathrm{P}=0.005)$, elevated AFP levels $(\mathrm{P}=0.003)$, and presence of tumor thrombus $(\mathrm{P}=0.025)$ (Table 2), while low NRF2 expression was associated with larger tumor size $(\mathrm{P}=0.022)$ (Table 3). A further investigation also showed that elevated AFP levels directly influenced long-term OS $(\mathrm{HR}=1.595 ; 95 \% \mathrm{CI}, 0.997-2.551, \mathrm{P}=0.051)$ and DFS (HR=1.495; 95\% CI, 1.007-2.221, $\mathrm{P}=0.046)$. Presence of tumor
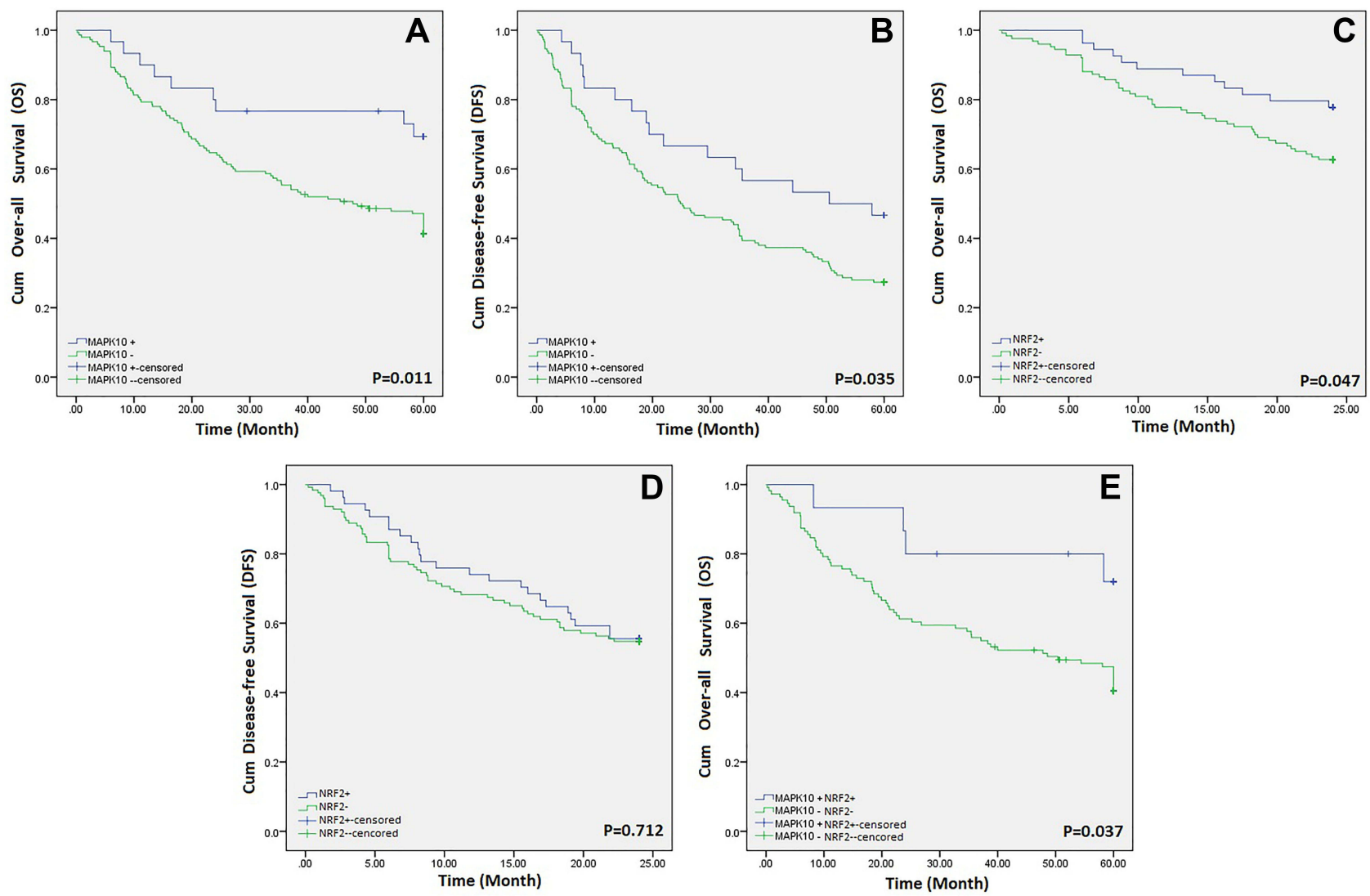

Figure 2 Survival curves of HCC patients based on MAPK 10 and NRF2 expression levels. (A) long-term overall survival curves of high and low MAPK I0 expression groups. (B) long-term disease-free survival curves of high and low MAPKI0 expression groups. (C) short-term overall survival curves of high and low NRF2 expression groups. (D) short-term disease-free survival curves of high and low NRF2 expression groups. (E) long-term overall survival curves of high and low MAPKI0 and NRF2 expression groups. 
Table 2 Clinical Characteristics of MAPKI0 Protein Expression Groups (18I Patients)

\begin{tabular}{|c|c|c|c|}
\hline$N(\%)(181)(100)$ & High Expression 30 (16.57) & Low Expression I5 I (83.43) & $\mathbf{P}$ \\
\hline Gender (M/F) & $25 / 5$ & $134 / 17$ & 0.602 \\
\hline $\mathrm{HBsAg}( \pm)$ & $26 / 4$ & $126 / 25$ & 0.867 \\
\hline Cirrhosis $( \pm)$ & $24 / 6$ & $128 / 23$ & 0.706 \\
\hline MVI ( $( \pm)$ & $6 / 24$ & $72 / 79$ & 0.005 \\
\hline$B C L C(0+A / B+C)$ & $19 / 11$ & $70 / 81$ & 0.089 \\
\hline Tumor differentiation $(|+| I / I I I+\mid \mathrm{V})$ & $21 / 9$ & $92 / 59$ & 0.349 \\
\hline AFP (Normal/Elevated) & $18 / 12$ & $48 / 103$ & 0.003 \\
\hline CEA (Normal/Elevated) & $29 / 1$ & $138 / 13$ & 0.539 \\
\hline CAI9-9 (Normal/ Elevated) & $25 / 5$ & $114 / 37$ & 0.353 \\
\hline Number of lesions (single/multiple) & $23 / 7$ & $128 / 23$ & 0.412 \\
\hline Tumor size $(\leq 5 \mathrm{~cm} />5 \mathrm{~cm})$ & $14 / 16$ & $73 / 78$ & 0.867 \\
\hline Encapsulation (yes/no) & $19 / 11$ & $81 / 70$ & 0.33 \\
\hline Tumor thrombus $*(180)$ (yes/no) & $0 / 30$ & $27 / 123$ & 0.025 \\
\hline ALT (Normal/Elevated) & $17 / 13$ & $88 / 63$ & 0.87 \\
\hline AST * (I68) (Normal/Elevated) & $18 / 9$ & $92 / 49$ & 0.887 \\
\hline ALP * (164) (Normal/Elevated) & $24 / 2$ & $120 / 18$ & 0.661 \\
\hline GGT * (164) (Normal/ Elevated) & $7 / 19$ & $46 / 92$ & 0.521 \\
\hline TB * (174) (Normal/ Elevated) & $28 / 0$ & $136 / 10$ & 0.325 \\
\hline DB $*(175)($ Normal/Elevated $)$ & $28 / 1$ & $130 / 16$ & 0.366 \\
\hline
\end{tabular}

Note: *Total available number of patients data.

Abbreviations: $\mathrm{HBsAg}$, hepatitis B surface antigen; MVI, microvascular invasion; BCLC, Barcelona clinic liver cancer staging; AFP, alpha-fetoprotein; CEA, carcinoembryonic antigen; CA19-9, carbohydrate antigen 19-9; ALT, alanine aminotransferase; AST, aspartate aminotransferase; ALP, alkaline phosphatase; GGT, gamma-glutamyltransferase; TB, total bilirubin; DB, direct bilirubin.

thrombus was associated with similar results (OS: $\mathrm{HR}=2.843 ; 95 \% \mathrm{CI}, 1.733-4.665, \mathrm{P} \leq 0.001$, DFS: $\mathrm{HR}=2.644 ; 95 \% \mathrm{CI}$, $1.662-4.205, \mathrm{P} \leq 0.001)$. Findings also showed that patients with smaller tumor lesions $(\leq 5 \mathrm{~cm})$ had prolonged OS (12 months) ( $\leq 5 \mathrm{~cm}: 46.99 \pm 2.138$ vs $>5 \mathrm{~cm}: 34.387 \pm 2.421, \mathrm{P}<0.001)$.

\section{Discussion}

The phenomenon of aging has always been a favorable topic in medical research. The rapid aging of worldwide populations ${ }^{1}$ and its influence on cancer incidence ${ }^{38}$ magnified the need to explore relevant mechanisms. However, the lack of age-specific research in cancer patients prevents relevant guidelines from considering the age as a factor when suggesting treatment strategies. Currently, a little is known about how well-known aging theories might be affected by the presence of cancer. For example, NRF2 functions, an oxidative stress regulator, decrease with age; however, studies found that NRF2 pathways can be hijacked by cancer cells to ensure survival and aggressiveness. ${ }^{8,13,22,23}$ Yet, the influence of age on NRF2 expression and related mechanisms in cancer patients is rarely addressed. Thus, this study's aim was to analyze the influence of age on NRF2 and MAPK10 (NRF2 activator) expression in HCC patients, investigate the age-specific features of NRF2 and MAPK10 interaction, and evaluate the clinical significance of relevant findings.

We started this study by investigating NRF2 expression in HCC and near-tumor samples and analyzing potential differences between age groups ( $\leq 59 \mathrm{y}$ vs $\geq 60$ y). Samples from 2009-2010 were included to insure the availability of 
Table 3 Clinical Characteristics of NRF2 Protein Expression Groups (I8I Patients)

\begin{tabular}{|c|c|c|c|}
\hline$N(\%)(181)(100)$ & High Expression 54 (29.83) & Low Expression 127 (70.17) & $\mathbf{P}$ \\
\hline Gender (M/F) & $45 / 9$ & $114 / 13$ & 0.226 \\
\hline $\mathrm{HBsAg}( \pm)$ & $44 / 10$ & $108 / 19$ & 0.550 \\
\hline Cirrhosis $( \pm)$ & $47 / 7$ & $105 / 22$ & 0.464 \\
\hline MVI $( \pm)$ & $25 / 29$ & $53 / 74$ & 0.571 \\
\hline$B C L C(0+A / B+C)$ & $24 / 30$ & $65 / 62$ & 0.407 \\
\hline Tumor differentiation $(|+| I / I I+\mid \mathrm{IV})$ & $32 / 22$ & $81 / 46$ & 0.566 \\
\hline AFP (Normal/ Elevated) & $14 / 40$ & $52 / 75$ & 0.055 \\
\hline CEA (Normal/ Elevated) & $49 / 5$ & $118 / 9$ & 0.844 \\
\hline CAI9-9 (Normal/ Elevated) & $46 / 8$ & $93 / 34$ & 0.081 \\
\hline Number of lesions (single/multiple) & $43 / 11$ & $108 / 19$ & 0.371 \\
\hline Tumor size $(\leq 5 />5)$ & $33 / 21$ & $54 / 73$ & 0.022 \\
\hline Encapsulation (yes/no) & $27 / 27$ & $73 / 54$ & 0.354 \\
\hline Tumor thrombus $*(180)$ (yes/no) & $7 / 47$ & $20 / 106$ & 0.616 \\
\hline ALT (Normal/Elevated) & $28 / 26$ & $77 / 50$ & 0.274 \\
\hline AST * (I68) (Normal/ Elevated) & $30 / 20$ & $80 / 38$ & 0.331 \\
\hline ALP $*(164)($ Normal/ Elevated) & $44 / 4$ & $100 / 16$ & 0.331 \\
\hline GGT * (164) (Normal/ Elevated) & $14 / 34$ & $39 / 77$ & 0.579 \\
\hline TB * (174) (Normal/ Elevated) & $48 / 3$ & $116 / 7$ & I \\
\hline DB * (I75) (Normal/ Elevated) & $44 / 8$ & $114 / 9$ & 0.100 \\
\hline
\end{tabular}

Note: $*$ The total available number of patients data.

Abbreviations: $\mathrm{HBsAg}$, hepatitis B surface antigen; MVI, microvascular invasion; BCLC, Barcelona clinic liver cancer staging; AFP, alpha-fetoprotein; CEA, carcinoembryonic antigen; CAI9-9, carbohydrate antigen 19-9; ALT, alanine aminotransferase; AST, aspartate aminotransferase; ALP, alkaline phosphatase; GGT, gamma-glutamyltransferase; TB, total bilirubin; DB, direct bilirubin.

long-term follow-up data. Initially, we wanted to choose $80 \mathrm{y}$ as a cut-off for age groups, considering the continuous change in life expectancy and relevant cut-off in age-specific researches. ${ }^{39,40}$ However, we had to lower the cut-off to $60 \mathrm{y}$ to address the limited number of elderly patients. Our findings showed that high NRF2 expression was more common in HCC group (29.83\%) compared to near-tumor group (11.38\%) $(\mathrm{P}<0.001)$ which indicates the correlation between HCC and NRF2 expression and falls in line with findings of other studies. ${ }^{30}$ However, similar results were only found in $\leq 59 \mathrm{y}$ group. That indicates that the strong influence of age on NRF2 expression should not be neglected as it can directly affect the significance of findings, and raises the question whether studies' age-standardized findings are applicable in elderly patients who are usually excluded from relevant research.

In MAPK10 analysis, findings showed lower rates of expression in HCC samples in both age groups and no agespecific differences were found. However, age-specific patterns were observed when analyzing the relation between MAPK10 and NRF2 expression. Our finding showed that higher MAPK10 expression significantly correlated with high NRF2 expression in $\leq 59$ y group ( $\mathrm{P}=0.011)$ but not in $\geq 60$ y group $(\mathrm{P}=0.515)$. Such findings in younger patients fall in line with MAPK10 known role of activating NRF2 expression ${ }^{17-21}$; however, they also show how the ability of MAPK10 to activate NRF2 expression declines with age and how findings of age-standard studies may not explain the specific mechanisms in elderly patients. Such differences between age groups can also explain our previous results of why NRF2 expression was significantly higher in younger patients and not the elderly group. 
The following section of our study investigated genetic mutations and mRNA expression patterns of MAPK10 in order to explain the previously mentioned IHC results. In this section, we used global databases such as TCGA and ICGC $^{31,32}$ in order to 1) include sufficient number of patients, 2) include as many elderly patients as possible, 3) and include worldwide patients. Results from HCC patients were compared to results from patients with colorectal cancer, stomach cancer, and pancreatic cancer. The choice of the three types of cancer (colorectal, stomach, and pancreatic cancer) was based on the availability of data and the number of patients. Our results showed higher mutation rates in pancreatic cancer $(28.43 \%)$ and HCC $(25.37 \%)$ groups. Such results emphasize the importance of understanding the role of MAPK10 in both cancers. HCC was the only cancer showing a gradual increase in mutation rates with age. In addition, an increase of $21.23 \%$ was observed between younger $(<60 \mathrm{y})$ and older $(\geq 60 \mathrm{y})$ HCC patients $(\mathrm{P}<0.001)$. Therefore, the influence of age on mutation rates is apparent. The results can be explained by the well-known accumulation of genetic mutations with age. Yet, the significant difference between older patients $(\geq 60 \mathrm{y})$ in $\mathrm{HCC}$ group and compare group $(17.78 \%(\mathrm{P}<0.001))$ suggests that HCC patients are more susceptible to such age-dependent mutagenesis. That magnifies the importance of considering the age of patients in the screening and treatment strategies for HCC patients. Our findings also showed that only colorectal cancer group had a gradual decrease with age. These results can explain the current changes in colorectal cancer incidence since an increase is found in younger patients $(\leq 50 \mathrm{y})$ while the incidence in elderly patients keeps on declining. ${ }^{41}$ The early-age mutagenesis can be explained by the unhealthy lifestyle habits in younger generations. For example, studies have linked red and processed meat consumption (more common in the diet of younger generations) to increased mutagenesis and microbiome alterations which can translate into inflammatory and carcinogenic changes that cause early-onset colorectal cancer. ${ }^{42-46}$ Findings of mRNA analysis revealed lower expression rates in older patients $(\mathrm{P}=0.003)$. Such results indicate a poorer survival in elderly patients since high MAPK10 expression is beneficial for survival.

To investigate the clinical significance of MAPK10 and NRF2 protein expression, we analyzed the influence of their expression levels on survival rates and clinical features. Long-term and short-term survivals were represented by 5-year and 2-year survival rates, respectively. The selection was based on clinical significance and cut-off values reported in other researches and guidelines. ${ }^{47-50}$ Our findings showed that high MAPK10 expression was significantly beneficial for long-term OS and DFS and prolonged patients' survival for 10 months. Such clinical significance emphasizes the importance of MAPK10 in HCC patients and shows how lower expression levels in HCC (as found in our IHC analysis) play a role in the poor prognosis of this cancer. Our analysis of clinical characteristics also supported these results, as low MAPK10 expression was significantly associated with higher rate of MVI ( $\mathrm{P}=0.005)$, elevated AFP levels $(\mathrm{P}=0.003)$, and presence of tumor thrombus $(\mathrm{P}=0.025)$. These clinical features directly affected OS and DFS of HCC patients. We also found that NRF2 high expression prolonged short-term OS for 2 months $(\mathrm{P}=0.047)$, While low NRF2 expression was associated with larger tumor size $(\mathrm{P}=0.022)$. Therefore, our previous findings of lower NRF2 expression rates in older patients are also very important when predicting patients' prognosis. The importance of MAPK10 expression and ability to stimulate NRF2 expression was also present in our findings, since high expression of both MAPK10 and NRF2 prolonged long-term OS for 13 months. In addition, patients with low MAPK10 expression showed no significant difference in survival based on NRF2 expression alone. Thus, the decrease in such ability with age is also clinically significant and should be addressed.

Challenges such as the retrospectivity and limited number of elderly patients included in some sections of the study forced certain limitations during the completion of this research. It mainly affected the selection of age groups cut-off. Nonetheless, our study presented important findings that demonstrated how the process of aging should not be neglected in HCC research and how excluding elderly patients from relevant researches compromised the application of general findings in these patients. Since our study focused on HCC patients and based our conclusion on clinical findings, further experimental investigation can help supporting our results, expand our knowledge regarding this topic, and explore relevant mechanisms on a cellular level.

This study presents age-specific differences in NRF2 expression patterns in HCC patients. It shows a significant decrease in expression with age, and links it to a potential decline in MAPK10 ability to activate NRF2 in older patients. Furthermore, our findings clarify the influence of such expression patterns on patients' survival and clinical characteristics. Therefore, we 
believe our findings help explaining the poor prognosis of $\mathrm{HCC}$ in elderly patients and form an important base to develop a health care system that considers the age of patients in its screening, diagnosis, and treatment plans.

\section{Conclusion}

NRF2 expression in HCC patients is influenced by age. Lower levels of expression are observed in older age groups due to a potential decline in MAPK10 ability to activate NRF2 in older patients. Such a decline in MAPK10 ability, suggested by our clinical findings, is accompanied with increased genetic mutation rates and decreased mRNA expression of this gene. MAPK10 and NRF2 expression levels influence the survival of patients as lower expression is associated with shorter survival and poorer prognosis due to positive correlation with microvascular invasion, tumor thrombus, elevated AFP levels, and larger tumor size.

\section{Consent for Publication}

All authors have read and approved the paper and declare no potential conflicts of interest in the paper. If the paper is accepted, all the authors will observe the terms of the License to Publish. All authors have agreed on the journal to which the article will be submitted, gave final approval of the version to be published, and agree to be accountable for all aspects of the work.

\section{Funding}

This work was supported by grants from the National Natural Science Foundation of China $(82073208,82103521)$, the Special Foundation for Science and Technology Basic Research Program (2019FY101103) the Shanghai Sailing Program (21YF1407500), the Shanghai Shen Kang Hospital Development Center new frontier technology joint project (SHDC12021109), and the China Postdoctoral Science Foundation (2021M690674).

\section{Disclosure}

The authors declare that they have no competing interests in this work.

\section{References}

1. United Nations, Department of Economic and Social Affairs, Population division. World Population Prospects 2019: highlights [Internet]; 2019 [cited August 21, 2019]. Available from: https://www.un.org/development/desa/publications/world-population-prospects-2019-highlights.html. Accessed January 15, 2022.

2. Pilleron S, Soto-perez-de-celis E, Vignat J, et al. Estimated global cancer incidence in the oldest adults in 2018 and projections to 2050. Int J Cancer. 2021;148(3):601-608. doi:10.1002/ijc.33232

3. Gouverneur A, Salvo F, Berdaï D, Moore N, Fourrier-Réglat A, Noize P. Inclusion of elderly or frail patients in randomized controlled trials of targeted therapies for the treatment of metastatic colorectal cancer: a systematic review. J Geriatr Oncol. 2018;9(1):15-23. doi:10.1016/j. jgo.2017.08.001

4. Bray F, Ferlay J, Soerjomataram I, Siegel RL, Torre LA, Jemal A. Global cancer statistics 2018: GLOBOCAN estimates of incidence and mortality worldwide for 36 cancers in 185 countries. CA Cancer J Clin. 2018;68(6):394-424. doi:10.3322/caac.21492

5. Zhang S, Zheng R, Zeng H, Chen W. [The incidence differences among sex, geographical areas and mean age of diagnosis for liver cancer in China, 1989-2008]. Zhonghua Yu Fang Yi Xue Za Zhi. 2014;48(5):355-360. in Chinese.

6. Akinyemiju T, Abera S, Ahmed M, et al.; Global Burden of Disease Liver Cancer Collaboration. The burden of primary liver cancer and underlying etiologies from 1990 to 2015 at the global, regional, and national level: results from the Global Burden of Disease Study 2015. JAMA Oncol. 2017;3(12):1683-1691. doi:10.1001/jamaoncol.2017.3055.

7. López-Otín C, Blasco MA, Partridge L, Serrano M, Kroemer G. The hallmarks of aging. Cell. 2013;153(6):1194-1217. doi:10.1016/j. cell.2013.05.039

8. Schmidlin CJ, Dodson MB, Madhavan L, Zhang DD. Redox regulation by NRF2 in aging and disease. Free Radic Biol Med. 2019;134:702-707. doi:10.1016/j.freeradbiomed.2019.01.016

9. Lewis KN, Wason E, Edrey YH, Kristan DM, Nevo E, Buffenstein R. Regulation of Nrf2 signaling and longevity in naturally long-lived rodents. Proc Natl Acad Sci U S A. 2015;112(12):3722-3727. doi:10.1073/pnas.1417566112

10. Hayes JD, Dinkova-Kostova AT. The Nrf2 regulatory network provides an interface between redox and intermediary metabolism. Trends Biochem Sci. 2014;39(4):199-218. doi:10.1016/j.tibs.2014.02.002

11. Reddy NM, Kleeberger SR, Yamamoto M, et al. Genetic dissection of the Nrf2-dependent redox signaling-regulated transcriptional programs of cell proliferation and cytoprotection. Physiol Genomics. 2007;32(1):74-81. doi:10.1152/physiolgenomics.00126.2007

12. Jaiswal AK. Regulation of genes encoding NAD(P)H: quinone oxidoreductases. Free Radic Biol Med. 2000;29(3-4):254-262. doi:10.1016/S08915849(00)00306-3

13. Kitamura H, Motohashi H. NRF2 addiction in cancer cells. Cancer Sci. 2018;109(4):900-911. doi:10.1111/cas.13537 
14. Satoh H, Moriguchi T, Taguchi K, et al. Nrf2-deficiency creates a responsive microenvironment for metastasis to the lung. Carcinogenesis. $2010 ; 31$ (10):1833-1843. doi:10.1093/carcin/bgq105

15. Hiramoto K, Satoh H, Suzuki T, et al. Myeloid lineage-specific deletion of antioxidant system enhances tumor metastasis. Cancer Prev Res. 2014; 7 (8):835-844. doi:10.1158/1940-6207.CAPR-14-0094

16. Maj T, Wang W, Crespo J, et al. Oxidative stress controls regulatory T cell apoptosis and suppressor activity and PD-L1-blockade resistance in tumor. Nat Immunol. 2017;18(12):1332-1341. doi:10.1038/ni.3868

17. Slenter DN, Kutmon M, Hanspers K, et al. WikiPathways: a multifaceted pathway database bridging metabolomics to other omics research. Nucleic Acids Res. 2018;46(D1):D661-D667. doi:10.1093/nar/gkx1064

18. Martens M, Ammar A, Riutta A, et al. WikiPathways: connecting communities. Nucleic Acids Res. 2021;49(D1):D613-D621. doi:10.1093/nar/ gkaa1024

19. Bak MJ, Truong VL, Ko SY, et al. Induction of Nrf2/ARE-mediated cytoprotective genes by red ginseng oil through ASK1-MKK4/7-JNK and p38 MAPK signaling pathways in HepG2 cells. J Ginseng Res. 2016;40(4):423-430. doi:10.1016/j.jgr.2016.07.003

20. Tseng CK, Hsu SP, Lin CK, Wu YH, Lee JC, Young KC. Celastrol inhibits hepatitis C virus replication by upregulating heme oxygenase-1 via the JNK MAPK/Nrf2 pathway in human hepatoma cells. Antiviral Res. 2017;146:191-200. doi:10.1016/j.antiviral.2017.09.010

21. Jang HY, Hong OY, Chung EY, Park KH, Kim JS. Roles of JNK/Nrf2 pathway on hemin-induced heme oxygenase-1 activation in MCF-7 human breast cancer cells. Medicina. 2020;56(6):268. doi:10.3390/medicina56060268

22. Done AJ, Gage MJ, Nieto NC, Traustadóttir T. Exercise-induced Nrf2-signaling is impaired in aging. Free Radic Biol Med. 2016;96:130-138. doi:10.1016/j.freeradbiomed.2016.04.024

23. Suh JH, Shenvi SV, Dixon BM, et al. Decline in transcriptional activity of Nrf2 causes age-related loss of glutathione synthesis, which is reversible with lipoic acid. Proc Natl Acad Sci U S A. 2004;101(10):3381-3386. doi:10.1073/pnas.0400282101

24. Dodson M, de la Vega MR, Cholanians A, Schmidlin CJ, Chapman E, Zhang DD. Modulating NRF2 in disease: timing is everything. Annu Rev Pharmacol Toxicol. 2019;59(1):555-575. doi:10.1146/annurev-pharmtox-010818-021856

25. Romero R, Sayin VI, Davidson SM, et al. Keap1 loss promotes Kras-driven lung cancer and results in dependence on glutaminolysis. Nat Med. 2017;23(11):1362-1368. doi:10.1038/nm.4407

26. Shibata T, Kokubu A, Saito S, et al. NRF2 mutation confers malignant potential and resistance to chemoradiation therapy in advanced esophageal squamous cancer. Neoplasia. 2011;13(9):864-873. doi:10.1593/neo.11750

27. Martinez VD, Vucic EA, Thu KL, Pikor LA, Lam S, Lam WL. Disruption of KEAP1/CUL3/RBX1 E3-ubiquitin ligase complex components by multiple genetic mechanisms: association with poor prognosis in head and neck cancer. Head Neck. 2015;37(5):727-734. doi:10.1002/hed.23663

28. Raghunath A, Sundarraj K, Arfuso F, Sethi G, Perumal E. Dysregulation of Nrf2 in hepatocellular carcinoma: role in cancer progression and chemoresistance. Cancers. 2018;10(12):481. doi:10.3390/cancers 10120481

29. Cheng ML, Lu YF, Chen H, Shen ZY, Liu J. Liver expression of Nrf2-related genes in different liver diseases. Hepatobiliary Pancreat Dis Int. 2015;14(5):485-491. doi:10.1016/S1499-3872(15)60425-8

30. Zhang M, Zhang C, Zhang L, et al. Nrf2 is a potential prognostic marker and promotes proliferation and invasion in human hepatocellular carcinoma. BMC Cancer. 2015;15(1):531. doi:10.1186/s12885-015-1541-1

31. Zhang J, Bajari R, Andric D, et al. The international cancer genome consortium data portal. Nat Biotechnol. 2019;37(4):367-369. doi:10.1038/ s41587-019-0055-9

32. Zhang J, Baran J, Cros A, et al. International cancer genome consortium data portal-a one-stop shop for cancer genomics data. Database. 2011;2011:bar026. doi:10.1093/database/bar026

33. Gao J, Aksoy BA, Dogrusoz U, et al. Integrative analysis of complex cancer genomics and clinical profiles using the cBioPortal. Sci Signal. 2013;6 (269):pl1. doi:10.1126/scisignal.2004088

34. Cerami E, Gao J, Dogrusoz U, et al. The cBio cancer genomics portal: an open platform for exploring multidimensional cancer genomics data. Cancer Discov. 2012;2(5):401-404. doi:10.1158/2159-8290.CD-12-0095

35. Uhlén M, Fagerberg L, Hallström BM, et al. Proteomics. Tissue-based map of the human proteome. Science. 2015;347(6220):1260419. doi:10.1126/science. 1260419

36. Uhlen M, Zhang C, Lee S, et al. A pathology atlas of the human cancer transcriptome. Science. 2017;357(6352):eaan2507. doi:10.1126/science. aan 2507

37. $\mathrm{Hu} \mathrm{B}, \mathrm{Ma} \mathrm{X}, \mathrm{Fu} \mathrm{P}$, et al. miRNA-mRNA regulatory network and factors associated with prediction of prognosis in hepatocellular carcinoma Genomics Proteomics Bioinformatics. 2021. doi:10.1016/j.gpb.2021.03.001

38. Wu CC, Li MN, Meng HB, et al. Analysis of status and countermeasures of cancer incidence and mortality in China. Sci China Life Sci. 2019;62 (5):640-647. doi:10.1007/s11427-018-9461-5

39. Nozawa A, Kubo S, Takemura S, et al. Hepatic resection for hepatocellular carcinoma in super-elderly patients aged 80 years and older in the first decade of the 21st century. Surg Today. 2015;45(7):851-857. doi:10.1007/s00595-014-0994-1

40. Oishi K, Itamoto T, Kohashi T, Matsugu Y, Nakahara H, Kitamoto M. Safety of hepatectomy for elderly patients with hepatocellular carcinoma. World J Gastroenterol. 2014;20(41):15028-15036. doi:10.3748/wjg.v20.i41.15028

41. Mauri G, Sartore-Bianchi A, Russo AG, Marsoni S, Bardelli A, Siena S. Early-onset colorectal cancer in young individuals. Mol Oncol. 2019;13 (2):109-131. doi:10.1002/1878-0261.12417

42. The Lancet Oncology. Colorectal cancer: a disease of the young? Lancet Oncol. 2017;18(4):413.

43. Patel SG, Ahnen DJ. Colorectal cancer in the young. Curr Gastroenterol Rep. 2018;20(4):15. doi:10.1007/s11894-018-0618-9

44. Inra JA, Syngal S. Colorectal cancer in young adults. Dig Dis Sci. 2015;60(3):722-733. doi:10.1007/s10620-014-3464-0

45. Song M, Chan AT. Environmental factors, gut microbiota, and colorectal cancer prevention. Clin Gastroenterol Hepatol. 2019;17(2):275-289. doi:10.1016/j.cgh.2018.07.012

46. Murphy CC. Colorectal cancer in the young: Does screening make sense? Curr Gastroenterol Rep. 2019;21(7):28. doi:10.1007/s11894-019-0695-4

47. Poon RT, Fan ST, Lo CM, Liu CL, Wong J. Long-term survival and pattern of recurrence after resection of small hepatocellular carcinoma in patients with preserved liver function: implications for a strategy of salvage transplantation. Ann Surg. 2002;235(3):373-382. doi:10.1097/ 00000658-200203000-00009 
48. Ng KK, Vauthey JN, Pawlik TM, et al.; International Cooperative Study Group on Hepatocellular Carcinoma. Is hepatic resection for large or multinodular hepatocellular carcinoma justified? Results from a multi-institutional database. Ann Surg Oncol. 2005;12(5):364-373. doi:10.1245/ ASO.2005.06.004

49. European Association for the Study of the Liver. Electronic address: easloffice@easloffice.eu; European Association for the Study of the Liver. EASL Clinical Practice Guidelines: management of hepatocellular carcinoma. J Hepatol. 2018;69(1):182-236. doi:10.1016/j.jhep.2018.03.019

50. Heimbach JK, Kulik LM, Finn RS, et al. AASLD guidelines for the treatment of hepatocellular carcinoma. Hepatology. 2018;67(1):358-380. doi:10.1002/hep.29086

\section{Publish your work in this journal}

The International Journal of General Medicine is an international, peer-reviewed open-access journal that focuses on general and internal medicine, pathogenesis, epidemiology, diagnosis, monitoring and treatment protocols. The journal is characterized by the rapid reporting of reviews, original research and clinical studies across all disease areas. The manuscript management system is completely online and includes a very quick and fair peer-review system, which is all easy to use. Visit http://www.dovepress.com/testimonials.php to read real quotes from published authors.

Submit your manuscript here: https://www.dovepress.com/international-journal-of-general-medicine-journal 